\title{
EL ROL DE LA MUJER EN LA IGLESIA DE JESUCRISTO DE LOS SANTOS DE LOS ÚLTIMOS DÍAS
}

\section{The Role of Women in the Church of Jesus Christ of Latter-day Saints}

\author{
Nuccia Seminario Hurtado \\ J. Reuben Clark. \\ Capítulo Tuxtla Gutiérrez- México \\ México - DF México \\ https://orcid.org/0000-0002-1805-7780 \\ nuccia_sh@hotmail.com
}

\begin{abstract}
RESUMEN
El presente artículo aborda el rol y la historia que cumple la mujer miembro de la Iglesia de Jesucristo de los Santos de los Últimos Días desde una perspectiva social y teológica, así como también sus respectivas funciones y responsabilidades dentro de la asignación del Plan de Divino de nuestro Padre Celestial. Las mujeres santos de los últimos días se han visto en la necesidad de agruparse en una organización oficial y reconocida por la Iglesia para desarrollar y estudiar sus dones y atributos en su haber.
\end{abstract}

\section{Palabras clave:}

Mujer, Iglesia, Mormón, Divinidad y Sociedad de Socorro, Teología, fe.

\begin{abstract}
This article addresses the role and history of the female member of the Church of Jesus Christ of Latter-day Saints from a social and theological perspective, as well as their respective roles and responsibilities within the assignment of the Divine Plan of our celestial father. Latter-day saint women have found it necessary to group together in an official organization recognized by the Church to develop and study their gifts and attributes to their credit.
\end{abstract}

\section{Keywords:}

Woman, Church, Mormon, Divinity and Relief Society, Theology, Faith. 


\section{INTRODUCCIÓN}

"El mundo tiene suficientes mujeres duras; necesitamos mujeres delicadas. Hay suficientes mujeres groseras; necesitamos mujeres amables. Hay suficientes mujeres rudas; necesitamos mujeres refinadas. Hay suficientes mujeres que tienen fama $y$ dinero; necesitamos más mujeres que tengan fe. Hay suficiente codicia; necesitamos más abnegación. Hay suficiente vanidad; necesitamos más virtud. Hay suficiente popularidad; necesitamos más pureza" (La Iglesia de Jesucristo de los Santos de los Últimos Días. "El regocijo de ser mujer", Liahona, enero de 2001)

La Iglesia de Jesucristo de los Santos de los Últimos Días también conocida como "la corriente del mormonismo" posee alrededor de 14 millones de integrantes en todo el mundo, $\mathrm{y}$, se estima que están construyendo 145 templos aproximadamente. Esta religión posee 54,000 jóvenes de ambos sexos entre 18 a 25 años que se encuentran sirviendo una misión a tiempo completo con la finalidad de expandir su doctrina y su evangelio.

Esta religión tiene como fundamento esencial que las familias pueden ser eternas, quiere decir que tanto el hombre como la mujer deciden casarse en el Santo Templo mormón por este tiempo y las eternidades. No existe la figura de "hasta la muerte los separe", ya que se realizan sagradas alianzas y convenios celestiales en la Santidad del Señor para siempre $^{1}$, para ello, se requiere una ardua preparación temporal y espiritual entre los conyugues, especialmente en las mujeres, quienes actúan como ayudas idóneas de los varones.

En este presente artículo abordaremos el rol que cumple la mujer de la Iglesia de Jesucristo de los Santos de los Últimos Días, para así encontrar elementos esenciales que permitirán ahondar en sus cualidades, dones y atributos divinos, recurriendo a fuentes oficiales de la Iglesia y artículos científicos pertinentes, donde se podrá analizar desde un punto de vista teológico, sociológico y doctrinario.

\section{Las mujeres en el Libro de Mormón}

El Libro de Mormón es uno de los otros testamentos de Jesucristo. Es un libro canónico escrito en las Américas, descubierto y traducido por José Smith, el primer presidente de la Iglesia de Jesucristo de los Santos de los últimos Días. Los acontecimientos inician cuando una noche se le presentó el ángel Moroni, quien le reveló que las planchas de oro estaban cerca de una colina; con inspiración del Espíritu Santo y la bendición de Dios, José Smith pudo encontrarlas, y comenzar con su respectiva traducción al inglés.

La introducción del Libro de Mormón describe que este "es un volumen de escritura sagrada semejante a la Biblia. Es una historia de la comunicación de Dios con antiguos habitantes de las Américas y contiene la plenitud del Evangelio eterno." 2 Este libro fue escrito por los profetas vivientes durante los años 600 a.C. hasta 421 d.C. mediante la fe, la

1 Entiéndase a la Santidad del Señor como el Santo Templo, donde nos permiten "organizaos; preparad todo lo que fuere necesario; y estableced una casa, sí, una casa de oración, una casa de ayuno, una casa de fe, una casa de instrucción, una casa de gloria, una casa de orden, una casa de Dios." (Doctrina y Convenios 88:119)

2 Introducción descrita en el Libro de Mormón. 
revelación y el espíritu de profecía. (La Iglesia de Jesucristo de los Santos de los Últimos Días, 2020)

Aunado a ello, este libro es una recopilación de todos los hechos que se interrelacionan con la restauración de los profetas y enseñanzas del Evangelio vividas en las Américas, que nos proporciona información valiosa de mujeres que marcaron un hito en la actividad religiosa tras ser reconocidas como ayudas idóneas de los profetas, buenas madres y ejemplos de misioneras.

En primer lugar, comentamos sobre Saríah, esposa de Lehi ${ }^{3}$. El Señor advierte a Lehi que salga de la tierra de Jerusalén, porque este profetiza al pueblo sobre su iniquidad, y tratan de quitarle la vida. Lehi viaja tres días por el desierto con su familia. (1 Nefi 1:1, Libro de Mormón) Saríah acompañó a su esposo durante todo el trayecto en el desierto para llegar a la Américas, junto a sus hijos Lamán, Lemuel, Sam y Nefi, cumplieron la promesa del Señor. Saríah se caracteriza como una mujer fiel y de fe, porque cumplió con el designio de Dios, a pesar de su avanzada edad, fue una ayuda idónea de su conyugue Lehi en su propósito en la tierra.

La historia de Lehi, Saríah e hijos denota el amor sincero, la paciencia y la gratitud que trae frutos en la vida familiar. Se reconoce a Saríah como una mujer fiel a los mandamientos del Señor, todo ello se refleja en la toma de decisiones, tras sacrificar su hogar inicial y su familia para acompañar a su esposo a una nueva tierra prometida para que se cumpla el mandato del Señor. Asimismo, hemos de indicar que como ya hemos expresado en líneas anteriores, Saríah fue una mujer de avanzada edad, sin embargo, el Señor le dio la oportunidad de dar a luz a dos hijos en el desierto (José y Jacob), con la finalidad de henchir la tierra. (1 Nefi 1:1; $2: 1-5 ; 5: 1-9 ; 8: 14-16 ; 17: 1-2,55 ; 18: 7$, 17-19, Libro de Mormón)

Seguidamente, el libro de Alma ${ }^{4}$ describe a una mujer lamanita de fe, quien trabajó como sirvienta en la casa del Rey Lamoni e hizo un sinfín de milagros al compartir el Evangelio del Señor. La mujer se llamaba Abish. "Justo después de que Mormón escribió el nombre Abish (el padre es un hombre), inmediatamente siguió con el detalle de que "se había convertido al Señor muchos años antes a causa de una notable visión de su padre." (Alma 19:16, Libro de Mormón).

Adicionalmente, Abish ${ }^{5}$ en la historia de las escrituras, es considerada una misionera y seguidora fiel de Dios, porque se tuvo un proceso de conversión espiritual. Se describe que el "padre de Abish le enseñó a creer en Jesucristo (...) y que desde muy pequeña Abish supo que el Padre Celestial quería que ella compartiera el Evangelio con el pueblo. (La Iglesia de Jesucristo de los Santos de los últimos Días, 2016)

Por otro lado, existió otra mujer que también tuvo obras buenas en el Libro de Mormón. La esposa del rey Lamoni ${ }^{6}$, quien cumplió un rol crucial en su progreso porque fue la principal defensora y actora en su proceso de arrepentimiento y

3 Primer profeta en llegar a las Américas en el año 600 a.C. tras recibir una revelación de Dios según detallan las escrituras en el Libro de Mormón. (1 Nefi)

4 Libro canónico perteneciente al Libro de Mormón.

5 Abish fue una mujer lamanita que vivió hasta los 90 años según describen las escrituras.

6 Fue un rey en las antiguas Américas, se describe su rol en el libro de Alma en el Libro de Mormón. 
conversión. Las escrituras relatan que el rey Lamoni escuchó a Ammón ${ }^{7}$ predicar el Evangelio, y este fue consciente de su estado espiritual, el cual pidió de rodillas a Dios que pudiera cambiar su personalidad pecaminosa, entonces cayó sobre una cama. La esposa del rey Lamoni al verlo, acudió inmediatamente a Ammón a preguntarle qué había sucedido, pues sentía en su corazón que no había muerto. (Alma 18:40 43, Libro de Mormón).

En estos sucesos, se describe la firmeza y gratitud de la esposa del rey Lamoni, tras asistirlo durante todo su proceso de conversión con un corazón humilde y sincero, debido a que hubo malos comentarios de la actitud del rey durante su cambio, por ende, ella siempre creyó en su conyugue y lo impulsó a que realmente cambie de corazón, sin duda, un ejemplo loable de mujer fiel y seguidora del Evangelio.

Las tres mujeres tuvieron cualidades divinas y cumplieron un propósito en la tierra. La historia de las mujeres en el Libro de Mormón, enmarca un principio fundamental en los últimos días, que sus atributos y dones son especiales ante los ojos del Señor, debido a la valentía, coraje y esfuerzo de ser ayudas idóneas de los conyugues y fieles misioneras. En ese sentido, a continuación, un cuadro descriptivo de las cualidades esenciales de estas:

Tabla 1 Descripción de las mujeres en el Libro de Mormón

Mujer

Descripción y cualidad

- Mujer de fe

- Sacrificadora de bienes materiales

Saríah

- Ayuda idónea de su conyugue

- Predicadora fiel

Abish

- Misionera recta

- Mujer valiente
Véase en el Libro de Mormón
8:14-16; 17:1-2, 55; 18:7,

$17-19$

Alma 19-20
Esposa del

Rey

Lamoni
- Mujer de abundante fe

- Sierva firme del Señor

- Corazón humilde y sincero

Cuadro: Elaboración propia

Fuente: El Libro de Mormón

En estas descripciones, estas mujeres en el Libro de Mormón poseen cualidades que describen la divinidad que Dios da a sus hijas para que cumplan un rol y propósito en la tierra. Así como las mujeres en el Libro de Mormón cumplieron un designio importante al lado de sus esposos, también las mujeres

7 Sustraído de la guía para el estudio de las escrituras del Libro de Mormón: En el Libro de Mormón, el hijo del rey Mosíah. Ammón fue un misionero cuyos esfuerzos diligentes dieron como resultado la conversión de muchas almas a Cristo. 
actuales de la Iglesia de los Santos de los Últimos ${ }^{8}$ poseen funciones divinas que abordaremos en el siguiente acápite.

\section{Las funciones y responsabilidades divinas de la mujer}

La mujer en la Iglesia de Jesucristo de los Santos de los Últimos días cree en la importancia del rol dentro de la familia, en el matrimonio y en la maternidad. Los profetas vivientes describen que la mujer "es el adorno divino del género humano, que se expresa en... su capacidad para amar, su espiritualidad, delicadeza, resplandor, sensibilidad, creatividad, encanto, refinamiento, ternura, dignidad y serena fuerza. Se manifiesta en forma diferente en cada jovencita o mujer, pero todas la poseen. (La Iglesia de Jesucristo de los Santos de los Últimos Días, 2000, p.18)

El presidente Gordon B. Hinckley ${ }^{9}$ en la reunión general de la Sociedad de Socorro de septiembre de 1995, expresó que en el acto de la Familia: Una Proclamación para el Mundo es significativo porque permite presentar esa importante proclamación a las hermanas de la Iglesia. Al hacerlo, el presidente Hinckley enfatizó la influencia irremplazable de las mujeres en el plan del Señor dentro de sus roles como hijas de Dios.

Dentro de las enseñanzas sagradas se les indica que deben amar a Dios sobre todas las cosas en este tiempo y por las eternidades, preparándose para cumplir un rol en estos últimos días como esposas, madres y amas del hogar con la finalidad de cumplir el designio divino de Dios. En coherencia con el presidente Ezra Taft Benson ${ }^{10}$ quien mencionó que la obra divina de las mujeres tiene que ver con ser compañeras, cuidar del hogar y ser madres" (s/f. p. 64)

La familia: una proclamación para el mundo ${ }^{11}$ hace hincapié en que el primer mandamiento que Dios les dio a Adán y a Eva tenía que ver con el potencial que, como esposo y esposa, tenían de ser padres, en ese sentido "el esposo y la esposa tienen la solemne responsabilidad de amarse y cuidarse el uno al otro, y también a sus hijos. 'He aquí, herencia de Jehová son los hijos." (Salmos 127:3, La Santa Biblia)

En ese sentido, las mujeres santos de los últimos días cumplen una representación divina aquí en la tierra como en el Reino Celestial, sus funciones divinas permiten que pueda existir la procreación. Las sagradas escrituras expresan que Dios ha dicho que se debe, "Fructificad y multiplicaos, henchid la tierra y sojuzgadla; y tened dominio sobre los peces del mar, y sobre las aves del cielo, y sobre todo ser viviente que se mueve sobre la tierra." (Moisés 2:28, La Perla de Gran Precio)

Sin embargo, las funciones divinas de las mujeres de la Iglesia, no solamente se limitan a ser buenas esposas, madres e hijas, sino también con cumplir fielmente los mandamientos, doctrinas y principios de la Iglesia de Jesucristo de los Santos de los Últimos Días y enseñarles a sus hijos la plenitud del Evangelio. Los líderes de

8 También conocidas como "mormonas".

9 Decimoquinto presidente de la Iglesia de Jesucristo de los Santos de los Últimos Días (1995-2008).

10 Decimotercer presidente de la Iglesia de Jesucristo de los Santos de los Últimos Días (1985- 1994).

11 El presidente Gordon B. Hinckley leyó esta proclamación como parte de su mensaje en la Reunión General de la Sociedad de Socorro, que se llevó a cabo el 23 de septiembre de 1995, en Salt Lake City, Utah, EE. UU. 
la Iglesia mormona han exhortado a las mujeres lo siguiente:

Críen a sus hijos en la luz y la verdad; enséñenles a orar cuando son pequeños; léanles las Escrituras aun cuando no entiendan todo lo que les lean. Enséñenles a pagar el diezmo y las ofrendas desde la primera vez que reciban dinero; permitan que esa práctica se convierta en un hábito en su vida. Enseñen a sus hijos a honrar a las mujeres. Enseñen a sus hijas a andar en la virtud. Acepten responsabilidades en la Iglesia, y confíen en que el Señor los pondrá a la altura de cualquier llamamiento que reciban. Su ejemplo establecerá un modelo para sus hijos. ${ }^{12}$

\section{La mujer y el poder del sacerdocio}

La Iglesia de Jesucristo de los Santos de los Últimos Días es la Iglesia del Señor, y Su Iglesia se gobierna por y a través de la autoridad del sacerdocio y las llaves del mismo. "Las llaves del sacerdocio son la autoridad que Dios ha dado a los líderes del sacerdocio para dirigir, controlar y gobernar el uso de Su sacerdocio en la tierra. El ejercicio de la autoridad del sacerdocio lo gobiernan aquellos que poseen sus llaves [y] tienen derecho a presidir y dirigir la Iglesia en una jurisdicción" (La Iglesia de Jesucristo de los Santos de los Últimos Días, 2010).

Si bien es cierto, el poder del sacerdocio es una función divina que se ha conferido a los hombres, desempeñando así funciones distintas de las mujeres; ambos son una ayuda mutua e idónea para desarrollar sus dones. Así lo expresa Ballard (2013) "así como una mujer no puede concebir un hijo sin el hombre, tampoco el hombre puede ejercer plenamente el poder del sacerdocio para establecer una familia eterna sin la mujer... En la perspectiva eterna, el hombre y la mujer comparten el poder procreador y el poder del sacerdocio" (p.19)

La relación de la mujer y el sacerdocio se refleja en las alianzas sagrados que realizan el género femenino que profesa la fe mormona, dichos convenios sagrados tales como un bautismo, una confirmación del Espíritu Santo, un casamiento religioso en el templo, bendición de salud u otro, únicamente puede ser conferido por un hombre digno poseedor del Sacerdocio que sea parte de la religión mormona. "Los recursos que ayudan a todos los miembros de la Iglesia a comprender la autoridad del sacerdocio y el poder del sacerdocio disponibles para las mujeres, lo que significan y la forma en que las mujeres pueden desarrollarse y crecer en el poder del sacerdocio a medida que guardan más plenamente los convenios sagrados" (Bingham, 2019).

Al dirigirse a las mujeres de la Iglesia durante la Conferencia General de octubre de 2019, el presidente Russell M. Nelson ${ }^{13}$ invitó a las mujeres a (...) que comprendan que la restauración del sacerdocio es tan relevante para ustedes como mujeres como lo es para cualquier hombre", dijo el presidente Nelson. "Toda mujer y todo hombre que hace convenios con Dios y los guarda, y que participa dignamente en las ordenanzas del sacerdocio, tiene acceso directo al poder de Dios [...]. Les ruego que estudien con espíritu de oración todas las verdades que puedan encontrar acerca

12 Véase "Permanezcan firmes frente a las asechanzas del mundo", pág. 115

13 Profeta actual de la Iglesia de Jesucristo de los Santos de los Últimas Días. 
del poder del sacerdocio". (Eyre, 2020).

En ese sentido, la relación de la mujer y el sacerdocio se aproxima cuando este le confiere un llamamiento dentro de la Iglesia de Jesucristo, y, la mujer pueda servir con fe y amor a los demás. Asimismo, cuando se le confiere el poder de testificar ante todos y todas, a través del servicio misional. En el caso de las mujeres, dicho servicio puede hacerse a tiempo completo durante un año y medio, siempre y cuando sean solteras y dignas, esto quiere decir que hayan cumplido fielmente con los mandamientos y se hayan preparado espiritualmente mediante la lectura diaria de los libros canónicos sagrados, hayan recibido el curso de preparación para el templo y tengan un testimonio ferviente del Libro de Mormón.

\section{La sociedad de Socorro como organización oficial de las mujeres de la Iglesia}

Las organizaciones propias de las mujeres en la Iglesia de Jesucristo de los Santos de los Últimas Días son las Mujeres Jóvenes y la Sociedad de Socorro. Esta última se dedica a realizar tareas de asistencia social para los miembros de la iglesia y la sociedad en general. Las mujeres no reciben la ordenación sacerdotal, pero pueden ser profesoras, misioneras, bibliotecarias de capilla, directoras de música, directoras de comités de actividades, presidentas de la primaria, etcétera. (Zalpa, p.284)

La Sociedad de Socorro fue fundada por el profeta José Smith, el 17 de marzo de 1842 en Nauvoo, Illinois. La organización tenía dos objetivos principales: dar alivio a los pobres y a los necesitados, y ayudar a las personas a venir a Cristo. La Sociedad de Socorro continúa en la actualidad como una de las organizaciones de mujeres más grandes del mundo. Las hermanas se reúnen los domingos y en otras ocasiones y lugares según sea necesario. (La Iglesia de Jesucristo de los Santos de los Últimos Días, 2018)

Aunado a ello, la organización tiene como pilastra el reforzar las responsabilidades y funciones divinas que debe poseer una mujer en estos últimos días. "Dentro de la doctrina manejada por el grupo se hace énfasis en la revaloración de la mujer como persona y en su constante preparación para que se desempeñe como una buena madre, ama de casa y esposa. Estas enseñanzas se imparten por medio de una organización para señoras denominada Sociedad del Socorro, donde se les instruye sobre la importancia que las mujeres tienen el plan de Dios como madres, hijas, esposas, vecinas, compañeras de trabajo, ciudadanas, etcétera." (Juárez, 2000, p.86)

Los líderes de la iglesia mormona han manifestado que la organización de la SOCIEDAD DE SOCORRO, es una de las prioritarias porque permite que todas las mujeres mormonas puedan realizar la obra del Señor. "El presidente Smith explicó: '[El Señor] les ha dado esta gran organización en la que ellas tienen la autoridad para servir bajo la dirección de los obispos de los barrios... procurando el bienestar tanto espiritual como temporal de nuestro pueblo. ${ }^{14}$

La Sociedad de Socorro permite que las mujeres puedan reconocer sus talentos y dones con un afán de compartir con los demás (Kurton, 2016). Es una organización

14 Véase en el Discurso 'Relief Society_An Aid to the Priesthood'. 
inspirada por Dios, que solo en ella seremos capaces de emular el ejemplo de nuestro Salvador al seguir y poner en práctica nuestro lema. Sé que estamos en esta tierra para cumplir una misión de servicio y amor, que fuimos apartadas en la vida preterrenal por el Señor para alentar, amar y apoyar a nuestras hermanas en esta dispensación. Debemos vivir de tal manera que seamos dignas de pertenecer a la organización del Señor para la mujer. (Moncada, 2018)

Reafirmamos lo expresado por los lideres de la Iglesia de Jesucristo de los Santos de los Últimos Días en cuanto al propósito de la organización de la Sociedad de Socorro. Permite preparar a las mujeres en el ámbito espiritual y temporal mediante las enseñanzas de la fe y la familia. En ese sentido, el propósito de la Sociedad de Socorro es aumentar la fe, fortalecer las familias y servir al Señor. La Sociedad de Socorro es una hermandad que dura toda la vida. Todas las hermanas adultas, a partir de los dieciocho años, son miembros de la Sociedad de Socorro. Son miembros, aunque no puedan asistir a las reuniones de la Sociedad de Socorro.

\section{La mujer de la Iglesia de Jesucristo de los Santos de los Últimos Días en el ámbito educativo y temporal}

Hemos descrito las potencialidades divinas de cada mujer en estos últimos días, sin embargo, debemos evidenciar también sus funciones temporales y académicas en la actualidad. (BYU, 1878). La educación académica es parte del plan de nuestro Padre Celestial, ya que debemos educarnos y cultivar dones, talentos y actitudes para la vida terrenal y venidera. A propósito de ello, Dios ha mandado en estos últimos tiempos, a que podamos adquirir conocimiento secular para desempeñar un rol de mujeres emprendedoras y profesionistas, para de esa manera, contribuir en el hogar y ser las maestras de nuestros hijos.

Los miembros de la Iglesia de Jesucristo de los Santos de los Últimos Días, creen en la educación como un mandamiento esencial para adquirir competencias divinas y celestiales que les permitan asemejarse a Jesucristo. En esa misma línea, la religión enseña que deben tratar de aprender por el Espíritu y que tienen la responsabilidad de emplear su conocimiento para el bien de la humanidad. (La Iglesia de Jesucristo de los Santos de los Últimos Días, 2010), en relación y coherencia a lo que afirma el Presidente John Taylor ${ }^{15}$ quien en el año 1878 expresó lo siguiente: "El Señor nos ha mandado adquirir conocimiento tanto por el estudio como por la fe, y buscarlo en los mejores libros. Es nuestro deber enseñar a nuestros hijos y proporcionarles instrucción en todos los ramos de la educación que fomenten su bienestar", en ese sentido, las mujeres actúan diligentemente para adquirir una preparación académica para fortalecer la crianza de sus hijos.

Sin embargo, existen doctrinas que indican que las mujeres mormonas no deben estudiar ni trabajar, solamente ser esposas y madres, no obstante, nos permitimos incidir en que los profetas han enseñado que en estos últimos días todo el género femenino debe estar preparado en caso suceda algo en el hogar. "El tener estudios es la clave de la oportunidad económica. El Señor impuso a Su pueblo

15 Líder religioso nacido en Inglaterra. Se desempeñó como el tercer presidente de La Iglesia de Jesucristo de los Santos de los Últimos Días de 1880 a 1887. 
el mandato de buscar conocimiento 'tanto por el estudio como por la fe' (Doctrinas y Convenios 109:14). No hay duda de que podrán mantener mejor a su familia si tienen la mente y las manos capacitadas para hacer algo que sea remunerativo en la sociedad de la que van a formar parte.

Luego de todo lo descrito, se debe esclarecer que las mujeres santos de los últimos días deben estudiar y progresar desarrollando sus talentos en estos tiempos. No obstante, todo lo adquirido debe darse en beneficio de potenciar sus habilidades como mujer, esposa y madre, debido a que se necesita mujeres preparadas espiritualmente y temporalmente para que críen a sus hijos, y sean un ejemplo Divino. En coherencia con el Presidente Uchtdorf ${ }^{16}$, menciona que "para los miembros de la Iglesia, la educación no es simplemente una buena idea, sino un mandamiento. Hemos de aprender de cosas tanto en el cielo como en la tierra, y debajo de la tierra; cosas que han sido, que son y que pronto han de acontecer; cosas que existen en el país, cosas que existen en el extranjero." (Uchtdorf, 2009).

\section{CONCLUSIONES}

Las mujeres santos de los últimos días, también conocidas como "mormonas", poseen cualidades divinas que les permiten desarrollar atributos especiales y espirituales para ser buenas esposas, madres e hijas en este tiempo y en el mundo venidero.

Las mujeres mormonas también poseen cualidades, dones y talentos seculares y/o temporales que les permiten desarrollar sus capacidades cognitivas, a efectos de hacer uso de dichas habilidades em beneficio de su progreso personal y familiar.

Las mujeres mormonas, actúan como ayudas idóneas de sus conyugues en estos últimos días, quienes mediante sus dones y talentos magnifican el poder del sacerdocio y su rol de madres y esposas.

La Sociedad de Socorro, es una organización reconocida y oficial de la Iglesia de Jesucristo de los Santos de los Últimos Días, que cuenta con la participación activa de las mujeres, quienes potencian sus cualidades espirituales y temporales en su proceso de conversión espiritual.

El papel que cumple las mujeres santos de los últimos días es fundamental debido a que mediante a su desarrollo espiritual y temporal pueden alcanzar sus capacidades y competencias para lograr un pleno liderazgo dentro de la iglesia mormona, a efectos de fortalecer su rol como mujer, pero también familiar.

\section{REFERENCIAS BIBLIOGRÁFICAS}

Ballard, M. R. (2013). Esta es mi obra y mi gloria. Liahona 7 (5), 18-21.

Bingham. J. (2019). Investidas con poder. Conferencia de la Universidad Brigham Young para mujeres de mayo. womensconference.byu.edu

BYU. (1878). Deseret News: SemiWeekly. Utah: Brigham Young University.

16 Segundo consejero de la Primera Presidencia de La Iglesia de Jesucristo de los Santos de los Últimos Días. 
Eyring, H. (2020). Hermanas de Sión. Conferencia General de octubre. https://www.churchofjesuschrist. org/study/general-conference/202 0/10/35eyring?lang $=$ spa

Iglesia de Jesucristo de los Santos de los Últimos Días. (1982). Libro de Mormón. Otro testamento de Jesucristo. Buenos Aires, Argentina: Iglesia de Jesucristo de los Santos de los Últimos Días.

Iglesia de Jesucristo de los Santos de los Últimos Días. (2000). El regocijo del ser mujer: https://www. churchofjesuschrist.org/study/ general-conference/2000/10/thejoy-of-womanhood?lang $=$ spa

La Iglesia de Jesucristo de los Santos de los Últimos Días (2010). Manual de Administración de la Iglesia. Utah: La Iglesia de Jesucristo de los Santos de los Últimos Días.

La Iglesia de Jesucristo de los Santos de los Últimos Días (2016). Abish fue una misionera. https://www. churchofjesuschrist.org/study/ liahona/2016/06/children/abishwas-a-missionary?lang $=$ spa

La Iglesia de Jesucristo de los Santos de los Últimos Días. (2017). El propósito de la Sociedad de Socorro. Liahona 1(3), 18-21.

La Iglesia de Jesucristo de los Santos de los Últimos Días (2020). La traducción del Libro de Mormón. https://masfe.org/ creencias-santos-de-los-ultimosdias/libro-de-mormon/abish-librode-mormon/
Juárez, E. (2000). Recreando identidades en dos grupos religiosos. Revista de estudios de género, La ventana 1, 73-111.

Kurton, L. (2016). Sociedad de Socorro. https://noticias. laiglesiadejesucristo.org/articulo/ los-179-anos-de-la-sociedad-desocorro

MASFE. (2018). ¿Por qué Abish fue una de las únicas mujeres del Libro de Mormón en ser mencionada por su nombre?. https://masfe.org/ creencias-santos-de-los-ultimosdias/libro-de-mormon/abish-librode-mormon/

Moncada, K. (2018). Celebramos el aniversario de la Sociedad de Socorro inspirando a "venir a Cristo". https:// www.churchofjesuschrist.org/study/ liahona/2018/04/cea-local-news/ local-news -006 ?lang =spa

Uchtdorf, D. (2009). Dos principios para cualquier economía. https://www. churchofjesuschrist.org/study/ general-conference/2009/10/ tw o - principles - for - a n y economy?lang=spa

Zalpa, G. (s/f). La Iglesia de Jesucristo de los Santos de los Últimos Días en Aguascalientes. http://asociacionesreligiosas. gob.mx/work/models/ A s ociaciones Religiosas/ Resource/70/1/images/cap10.pdf

Fecha de recepción: 13/09/2021 Fecha de aceptación: 29/10/2021 